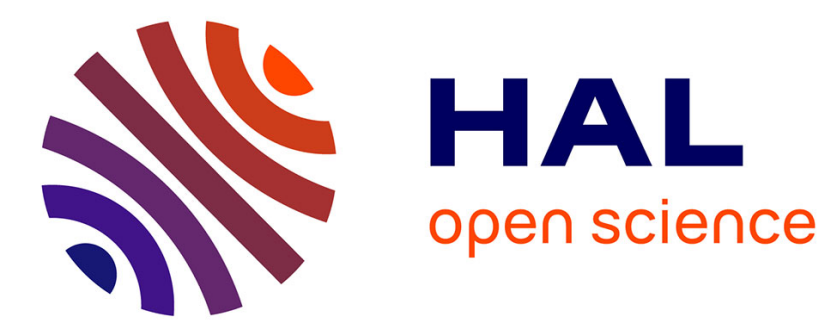

\title{
J. Butler et la fabrique discursive du sexe
}

Bruno Ambroise

\section{To cite this version:}

Bruno Ambroise. J. Butler et la fabrique discursive du sexe. Raisons politiques, 2003, 12 (12), pp.99-121. 10.3917/rai.012.0099 . halshs-00338157

\section{HAL Id: halshs-00338157 https://shs.hal.science/halshs-00338157}

Submitted on 15 Jan 2021

HAL is a multi-disciplinary open access archive for the deposit and dissemination of scientific research documents, whether they are published or not. The documents may come from teaching and research institutions in France or abroad, or from public or private research centers.

Copyright

L'archive ouverte pluridisciplinaire HAL, est destinée au dépôt et à la diffusion de documents scientifiques de niveau recherche, publiés ou non, émanant des établissements d'enseignement et de recherche français ou étrangers, des laboratoires publics ou privés. 


\section{J. Butler et la fabrique discursive du sexe.}

On ne nait pas femme, on le devient.

Simone de Beauvoir, L e D eux ième Sex e. Beaucoup aimeraient dire que l'homosex ualité et sa représentation culturelle ne sont pas dissociables, que la représentation ne s'ensuit pas de la sex ualité omme un pâle reflet, mais que la représentation a une fonction constitutive et que la sexualité s'ensuit de la représentation au moins omme un de ses effets.

Judith Butler, E x citable Speech, p. 123.

Théoricienne bien connue comme initiatrice de la Q ueer Theory, J. Butler est en train, par l'originalité de sa pensée et la radicalité de ses propos, de reprendre le flambeau du féminisme intellectuel et sa pensée devient désormais la référence obligée de toutes revendications portant sur les discriminations de sexe et de genre. Cette appropriation politique de la pensée de Butler, qui mélange allègrement et savamment des styles de pensé aussi différents que ceux de Hegel et Austin, Kripke et Derrida, Lacan et Foucault, ne va pas sans rencontrer quelques résistances ${ }^{1}$, mais semble inexorable tant elle devient reconnue internationalement. En témoigne la multiplicité des propos, interviews, articles d'elle actuellement publiés en France.

S'il ne s'agit pas de nier tout à la fois l'originalité philosophique de ses réflexions et l'importance stratégique de ses réflexions dans le champ politique contemporain, il convient plutôt de prendre au sérieux ses considérations philosophiques pour examiner leur pertinence et la portée de leur radicalité présumée. J. Butler adopte en effet un hyper-constructivisme et se propose de montrer que ce n'est pas seulement le genre qui est socialement construit, mais tout aussi bien le sexe - et qu'il est donc abusif de poser une frontière nette entre un genre qui cantonnerait les individus à tenir deux rôles précis et dotés d'attributions différentes et une réalité sexuelle supposée universelle et biologiquement fondée. En réalité, argumentera J. Butler, la division sexuelle fondée sur la biologie est tout autant une construction sociale que le partage des rôles homme/ femme. Il n'y aurait ainsi pas plus de distinction mâle/ femelle que de distinction entre les rôles d'homme et de femme fondée en nature'2. Il faudrait bien plutôt comprendre que la réalité sexuelle scindée entre un pôle mâle et un pôle femelle résulte elle-même d'une construction qu'elle appellera performative. Performative car cette construction réaliserait même ce dont elle parle, ou parce que les normes sexuelles ne seraient pas tant descriptives que prescriptives, pas tant régulatrices que constitutives, pour reprendre des termes de Searle ${ }^{3}$. On

\footnotetext{
${ }^{1}$.Voir l'article vengeur de sa "collègue" féministe M. Nussbaum, paru in T heN ew Republic, et traduit dans ce dossier.

2. Malgré leur inélégance, j'utiliserai dans ce texte les adjectifs "mâle" et "femelle" pour caractériser des attributs ou des pratiques corporels considérés comme naturels, les distinguant par là des attributs "féminins" et "masculins", considérés par la pensée féministe comme sociaux.

${ }^{3}$. Voir J.R. Searle, The Construction of Sodal Reality, Simon and Schuster, New Y ork, 1995.
} 
ne serait pas doté d'une sexualité masculine parce qu'on serait un être sexuel mâle, mais parce qu'on viserait à correspondre à l'idéal normatif de la masculinité, ce qui par là nous amènerait à réaliser une sexualité masculine. Le rôle définitionnel existerait ainsi préalablement à la réalité qu'il est censé définir et l'identité sexuelle propre à chacun ne serait que la réalisation différenciée qui, tout en se donnant pour naturelle, ne serait jamais que la réalisation d'une définition normative. Le rôle social construirait donc performativement la sexualité correspondante : il amènerait à la réalisation ce dont il dit rendre compte. J. Butler se sert ainsi d'un concept inauguré par J. L. Austin dans sa théorie des actes de parole, la performativité, pour dénaturaliser le sexe lui-même en tant que catégorie biologique et pour renverser le rapport de dépendance genre/ sexe : ce n'est plus le genre qui se base sur un sexe biologiquement donné, mais le sexe qui se construit en fonction d'un genre socialement assigné.

Mais, outre que l'on remarque immédiatement que J. Butler ne cesse de commettre une indistinction conceptuelle entre caractéristiques sexuelles et identité sexuelle, je voudrais remettre en cause son emploi très particulier du concept de performativité, qu'elle ne reprend pas tant, il est vrai, à A ustin qu'à un Austin retravaillé par D errida, mais qui, en tout état de cause, manque proprement la radicalité du concept de performativité pour lui faire jouer un rôle dans la radicalité d'une pensée qu'il ne peut pas jouer. Ainsi, je voudrais montrer qu'à trop considérer que la réalité sexuelle est déterminée par la parole, J. Butler en vient à oublier les conditions réelles et matérielles de la parole et de son efficace. Non pas donc que je veuille dire que les prétentions de son discours soient complètement illusoires, mais qu'elles le demeurent tant qu'elles ne se donnent pas les moyens de se mettre en oeuvre. A considérer que certaines de nos pratiques sont linguistiquement construites et déterminées, il faut considérer les conditions de possibilité de cette construction - et tout aussi bien ses limitations.

J'examinerai donc en premier lieu la construction théorique de J. Butler en ce qui concerne la contribution qu'apporterait le concept de performativité à la construction de l'identité sexuelle, puis ramènerait au jour la conceptualisation propre qu'en avait offert Austin et qui permet vraiment d'en cerner les possibilités et les limites. Je tâcherai alors de montrer qu'une compréhension réaliste - le propre de la pensée austinienne - du concept de performativité n'est pas sans conforter l'analyse de Butler sur certains points mais qu'elle la restreint sérieusement et lui donne des conditions que sa conceptualisation hyper-intellectualiste feint d'ignorer : j'essaierai ainsi de montrer qu'il n'est pas absurde de considérer que les identités sexuelles sont des réalités

\footnotetext{
${ }^{4}$. Voir J.L Austin, H ow To D o Things W ith W ords, edited by J.O. Urmson and M. Sbisà, Oxford University Press, «Oxford Paperbacks », Oxford, 2nde édition : 1976 ; trad. fr. de G. Lane, Q uand dire c'est faire, Editions du Seuil, coll. «L'ordre philosophique », Paris, 1970 ; réédité avec une postface de F. Récanati dans la coll. « Points-essais », 1991. Cité H .T.D . par la suite.
} 
performativement construites, mais en tant seulement qu'elles résultent de normes sociales qui satisfont des conditions très particulières de réussite - c'est-à-dire qui sont dotées du pouvoir, politique notamment, de s'imposer. Mais je contesterai que la parole, qu'elle soit performative ou non, puisse jamais construire la réalité sexuelle en tant que réalité biologique - tout simplement parce que cela supposerait des conditions de réussite que la performativité ne se donne pas, ne s'est jamais donnée et aura bien du mal à jamais se donner. Il conviendra donc de discerner chez Butler les acquis d'un constructivisme bien pensé et politiquement efficace parce que réaliste, des illusions déconstructionnistes qui tendent à lui faire prendre la corporéité même de la réalité sexuelle pour une construction idéologique naturalisée. Dire que le langage fait, en effet, ne revient pas à dire que ce qui est est langage. ${ }^{5}$

\section{La génération performative de la réalité sex uelle selon J. Butler.}

Avec Simone de Beauvoir et la publication du D euxième Sex e, la pensée féministe marqua une véritable rupture avec la pensée essentialiste en montrant que les rôles que tenaient les femmes et les hommes dans nos sociétés n'étaient pas liés à la nature respective de chacun, ni à une caractéristique ontologique propre, ni moins encore à un trait de caractère : les femmes ne s'occupaient pas des travaux ménagers parce qu'elles aimaient ça, étaient particulièrement douées pour la vaisselle ou parce que leur caractère fragile les éloignait des affres de la vie active ${ }^{6}$, mais tout simplement parce que l'organisation sociale leur intimait de ne pas s'occuper des affaires sérieuses, les reléguant par là à des rôles maternels et/ ou maternants. Dénaturalisant cette relégation qui se donnait comme partage normal (fondé en nature, voire en biologie) des tâches, la pensée féministe a donné essor à tout un ensemble de recherches sociologiques et/ ou anthropologiques qui ont confirmées ces thèses par des données nombreuses. C'est selon Butler ce qu'on peut montrer, par exemple, à partir des travaux de Levi-Strauss :

L'anthropologie structuraliste de Levi-Strauss qui inclut la problématique de la distinction entre nature et culture s'est vue appropriée par certaines théoriciennes féministes pour défendre et élucider la distinction entre le sexe et le genre : la position selon laquelle il y a une femelle naturelle ou biologique qui est transformé par après en "femme" socialement subordonnée, avec cette conséquence que le "sexe" est de l'ordre de la nature comme le "cru" et que le "genre" est de l'ordre de la culture comme le "cuit". (G ender Trouble, p. 47)

\footnotetext{
${ }^{5}$. J'ai bien conscience que j'adresse à Butler des reproches qu'on ne cesse de lui faire et auxquels elle ne cesse de répondre. Butler ne cesse ainsi de dire que la parole n'est pas le tout de la réalité, que le corps ne se réduit pas à la parole et que le sexe n'est pas littéraire (c'est même, dit-elle, la motivation de l'écriture de Bodies That M atter), mais elle ne s'en défend toujours que dans les préfaces et rien dans l'argumentation constituant le corps de sont texte ne peut le laisser présumer. Je me distinguerai des critiques habituels en attaquant Butler de l'intérieur de son argumentation, en remettant en question l'usage abusif qu'elle fait du concept de performativité.

${ }^{6}$. Au sens économique du terme.
} 
Même si elle va contester par après l'établissement de cette distinction, Butler dira que la construction du genre peut alors déjà être considérée comme performative :

le genre s'avère être performatif, c'est-à-dire qu'il constitue l'identité qu'il prétend être. [...] Il n'y a pas d'identité de genre derrière l'expression ; cette identité est performativement constituée par les "expressions" mêmes qu'on dit être son résultat. (G ender T rouble, $\mathrm{p}$. 33)

Pour Butler, qui est là proche des féministes "classiques", une signification est politiquement attribuée à des traits de personnalité qui les transforme en genre défini et acceptable. Dès lors, le genre est performatif en ce que "l'identité est [alors] un idéal normatif plutôt qu'un trait descriptif de l'expérience" : le rôle genré (homme ou femme) qui m'est attribué ne dérive ainsi pas de mon identité propre, je cherche seulement à le réaliser en cherchant à me conformer à une identité que la société me donne pour me définir. Dès lors, parce que je suis défini comme femme, je vais accomplir telle tâche, porter tels vêtements, pratiquer tels exercices, à l'exclusion de tels autres ; et c'est en accomplissant ce qui m'est ainsi prescrit que je réalise un genre alors considéré comme naturel.

Mais Butler va aller beaucoup plus loin que cette dénaturalisation du genre et, en s'inspirant de Foucault, essayer de montrer que le sexe lui-même résulte d'une construction sociale, puisqu'on ne trouve aucune distinction claire, ou du moins qui ne soit pas culturellement déterminée, entre les caractéristiques qui définiraient un sexe et celles qui seraient attribuées à un genre. En fait, la définition du sexe ellemême est tout sauf claire et pourrait bien s'avérer complètement artificielle et totalement arbitraire. Comme Foucault disait :

La notion de "sexe" rend possible de regrouper ensemble, selon une unité artificielle, des éléments anatomiques, des fonctions biologiques, des conduites, des sensations, et des plaisirs, et cela permettait qu'on fasse usage de cette unité fictive comme d'un principe causal, comme une signification omniprésente : le sexe était ainsi capable de fonctionner comme un signifiant unique et comme un signifié universel. (H istoire de la sex ualité, vol. 1)

Butler va reprendre cette idée que le sexe ainsi désigné n'est qu'une réalité fictive, mais une réalité fictive qui est performativement réalisée, c'est-à-dire portée au jour par les normes qui la définissent. Car elle retient cette idée de Foucault que le corps n'est pas sexué avant sa détermination à travers un discours au sein duquel il devient investi d'une idée de sexe naturel ou essentiel. C'est seulement après que ce discours a fait valoir sa norme qui devient sienne, que le corps cherche à se conformer à cette norme et réalise cette identité sexuelle : c'est alors seulement qu'il acquiert une identité en tant que sexe, ou devient corps sexué.

Cette réalisation de l'identité sexuelle va se faire au travers d'un certains mmbre de pratiques réitérées, déterminées par la norme, et qu'on va réifier sous une unité donnée comme identité sexuelle : ce n'est en fait pas notre sexualité déterminée qui nous pousse à certaines 
pratiques sexuelles, mais notre réalisation réitérée de ces pratiques qui nous confèrent une identité sexuelle précise. C'est ainsi parce que la société m'intime d'être un homme, et en tant que tel à avoir une sexualité masculine, que j'acquière une identité sexuelle de mâle en me construisant ma sexualité. Ce qui est premier, ce sont donc des pratiques déterminées par des normes sociales, et non pas une réalité sexuelle préalable à ces pratiques. A tel point que Butler va aller jusqu'à dire par moment que la réitération de certaines pratiques finit par constituer mon identité sexuée (et pas simplement sexuelle ; en fait, il semble que la distinction sexuelle/ sexuée ne soit pas si claire en anglais et Butler use et abuse de ce fait), quand elle dit par exemple qu'il s'agit de comprendre :

La construction du "sexe" non plus comme un donné corporel sur lequel le genre est artificiellement imposé, mais comme une norme culturelle qui gouverne la matérialisation des corps (the materialization of bodies) (Bodies That Matter, p. 2-3)

Tout cela conduit Butler à donner une première caractérisation de l'identité sexuelle comme réalisation performative.

De tels actes, gestes, etc., généralement réalisés, sont performatifs en ce sens que l'essence ou l'identité qu'ils visent autrement à exprimer sont des constructions fabriquées et soutenues à travers des signes corporels et d'autres moyens expressifs. Le fait que le corps genré soit performatif suggère qu'il n’a pas d'autre statut ontologique que les actes variés qui constituent sa réalité. (G ender Trouble, p. 173)

Si on considère en effet que le sujet sexuel (et donc son identité, voire son "essence" ) n'est jamais que le produit de ses actes, que la répétition de pratiques sexuelles qui l'identifient comme mâle ou comme femelle, alors on peut en effet considérer que l'identité sexuelle est performative en ce sens que ce sont des pratiques sexuelles définissant une certaine sexualité qui vont conférer cette identité sexuelle à la personne en question en amenant à la réalisation cette identité qui ne préexiste pas aux pratiques - tout comme la promesse ne préexiste pas à la pratique énonciatrice qui la fait et n'existe que par la grâce du pouvoir performatif de la parole. Dès lors, si l'identité sexuelle s'acquière bien par un processus de sexuation du sujet, alors les pratiques sexuelles auxquelles se livre une personne, en tant qu'elles sont déterminées par des normes présentes dans notre société, agissent bien comme définition performative de l'identité sexuelle de la personne qui les pratique. De même que la promesse n'est jamais qu'un énoncé de promesse, une identité sexuelle ne serait jamais que l'ensemble de pratiques qui répondent à la définition de cette identité. Bref, à faire certains actes sexuels hétérosexuels, je réponds à la définition de la sexualité masculine et me réalise donc comme être sexuel mâle, et à pratiquer d'autres actes sexuels hétérosexuels je réponds à la définition de la sexualité féminine et je me réalise comme femme. 
Ce que veut penser Butler, toutefois, ce n'est pas seulement le fait que l'identité sexuelle est une construction, car elle sent confusément que cela ne suffirait guère à qualifier cette réalité de "performative". Le fait que la succession d'actes amènent au jour une réalité n'est en effet guère autre chose que la caractéristique de tout acte. Auquel cas Butler n'aurait jamais mis au jour que le fait que la réalité sexuelle n'est pas une donnée naturelle mais une construction historique. Pour la penser comme performative, il faut bien voir que cette construction répond en plus à des normes définitionnelles (une réalité qui dit des choses) qui s'inscrivent dans les corps. Butler pose alors que ce qui est véritablement premier et détermine les pratiques qui constituent la réalité sexuelle, c'est le langage et ses normes.

la performativité doit être comprise, non pas comme un "acte" singulier ou délibéré, mais plutôt comme la pratique réitérative et citationnelle par laquelle le discours produit les effets qu'il nomme. Ce qui deviendra clair, j'espère, dans ce qui suit, c'est que les normes régulatrices $d u$ «sexe » travaillent sous un mode performatif pour constituer la matérialité des corps, et plus spécifiquement, pour matérialiser le sexe du corps, pour matérialiser la différence sexuelle en étant au service de la consolidation de l'impératif hétérosexuel ». (Bodies that Matter, p. 2.)

Il faut donc comprendre que les normes de la sexualité ont ce pouvoir performatif d'amener au jour ce dont elles parlent. Définissant la sexualité masculine, elles inscriraient en même temps celle-ci dans la réalité. Les normes sexuelles auraient donc le pouvoir de se mettre à exécution, ou plus exactement d'amener au jour la réalité qu'elles norment. Bien mieux, c'est parce qu'elles norment que la réalité normée adviendrait. Butler réinterprète ici la formule de Foucault selon laquelle les normes ne sont pas restrictives mais constructives, ce qui l'amène à penser le sujet comme normativité qu'il s'impose. Butler va penser l'identité sexuelle de façon similaire : elle n'est jamais qu'une réalité de normes mises au jour. Et sans l'existence de ces normes, il n'y aurait pas de réalité sexuelle.

Le "sexe" est toujours produit comme une réitération de normes hégémoniques. Cette réitération productive peut être lue comme une sorte de performativité. La performativité discursive semble produire cela qu'elle nomme, mettre au jour son propre référent, nommer et agir, nommer et faire. [...] Généralement, un performatif fonctionne en produisant cela qu'il déclare. [...] Aucun acte, en dehors d'une pratique régularisée et sanctionnée ne peut détenir le pouvoir de produire ce qu'il déclare. En effet, un acte performatif en dehors d'un ensemble de conventions réitérées et sanctionnées peut n’apparaitre que comme un vain effort de produire des effets qu'il ne peut pas produire. (Bodies That Matter, p. 107)

Butler considère ainsi que la sexualité réalisée est la réalisation performative de normes qui n'ont rien de naturel mais sont socialement construites et imposées, et que la réalité sexuelle advient de pratiques sexuelles qui ne reflètent jamais que la performativité des normes. La sexualité, dès lors, serait clairement un enjeu de pouvoir et ne serait que la réalisation de ce que le 
pouvoir (ou les mutliples instances de pouvoir) veut bien accepter. C'est même la raison pour laquelle les normes réussissent à s'inscrire dans la réalité en tant que sexualité - qui est par conséquent toujours sexualité légitime ou illégitime, conforme ou non à ces normes dont elle dérive. Le pouvoir dit en effet ce qu'est la sexualité, mais ce faisant, il ne dit pas ce qui est mais ce qu'il conçoit être comme sexualité normale. Comme tel, son discours est compris comme normatif et les corps vont chercher à y correspondre et vont par là adopter la sexualité correspondante à la définition que leur donne le pouvoir, en la réalisant par les pratiques correspondantes. Le pouvoir, parce qu'il est pouvoir, a les moyens de réaliser les normes qu'il pense comme naturelles et son discours va toujours tendre à créer ce qu'il dit. Lorsque le discours est "autorisé", pour reprendre une caractérisation de P. Bourdieu", la nomination qu'il effectue est toujours réalisation de ce qu'il dénomme. Son discours est donc par essence performatif et lorsqu'il concerne la sexualité , il réalise la sexualité dont il est parle. C'est ainsi que J. Butler en arrive à penser que, non seulement la sexualité est une construction historique, mais est aussi une réalisation performative : elle n'est jamais que le résultat d'un discours normatif qui amène au jour ce dont il parle. Tout corps sexué est ainsi le lieu de la réalisation d'un discours normatif et n'existe que par ces normes qui le forment et l'informent à la mode aristotélicienne. Le discours performatif concernant le sexe se traduit ainsi sous la forme de normes faites corps sexué. Par conséquent, le sexe masculin et le sexe féminin ne sont jamais que le résultat d'un discours hégémonique qui distingue (norme) deux identités sexuelles et qui les réalise en assignant à chaque corps des pratiques correspondantes.

Si la performativité réalise cette inscription des normes dans les corps en transformant les normes en corps, c'est parce que, on l'a vu, elle est autorisée, ou dotée de l'autorité nécessaire pour le faire. Or la performativité de la norme se traduit sous forme de pratiques sexuelles qui portent à la réalisation la norme à laquelle elles se conforment. Mais d'où vient le pouvoir qu'ont ces pratiques de construire une identité, c'est-à-dire de se stabiliser en un tout unitaire répondant à une norme ? Pourquoi le discours normatif at-il un tel pouvoir ? Pour Butler, la loi, et notamment la loi sexuelle, ne tire pas son efficacité des conventions sociales ou de l'autorité : ce serait faire reposer l'efficacité de la convention sociale qu'est la sexualité sur d'autre conventions sociales, or il s'agit bien plutôt expliquer cette efficacité ou cette autorité. Butler va donc faire appel à la caractérisation derridienne du pouvoir performatif. 8 Selon D errida, expliquer la réussite performative en en appelant à l'autorité qui la soutient n'explique rien car elle en appelle à l'autorité pour expliquer l'autorité. Il s'agit bien plutôt de comprendre d'où l'autorité tire sa force.

\footnotetext{
7. Voir P. Bourdieu, L anguage and Symbolic Power, Polity Press, Cambridge, 1992.

${ }^{8}$. Voir J. D errida, "Signature, événement, contexte", in L imited Inc., G alilée, Paris, 1990.
} 
En réalité, elle la tire du voilement de son absence d'origine par sa réitération constante d'un appel à l'autorité : le pouvoir n'est fondé sur rien d'autre qu'un appel à lui-même - par rien d'autre que par une auto-réréfence. Se prétendant fondé en autorité, il se fonde. D'une certaine façon, le pouvoir s'auto-fonde performativement en ne cessant de se faire valoir comme pouvoir. Cette répétition constante qui est donc la source du pouvoir, c'est ce que D errida va caractériser comme itérabilité. Et cette itérabilité sera aussi à la source de la performativité. La performativité n'est même qu'itérabilité, pouvoir constant de répétition, puisqu'il est tout à fait possible de caractériser la performativité comme auto-référentialité : un énoncé performatif réalise en effet ce à quoi il réfère, c'est-à-dire lui-même. Quand je dis "je promets", je n'en appelle selon Derrida qu'à rien d'autre qu'à mon énoncé pour réaliser la promesse : la promesse se réalise de par l'appel de l'énoncé à lui-même. C'est aussi ce que Derrida va appeler la citationnalité du discours performatif : le discours performatif n'est jamais que répétition citationnelle de lui-même et tire de là son pouvoir. Tel est pour Derrida, et pour Butler à sa suite, un trait structural du performatif.

Convaincue par cette idée, Butler va l'adapter à son discours et penser la réalisation performative de l'identité sexuelle en termes de citation - caractérisation linguistique censée valoir comme justification ultime de l'usage du terme de performatif. Les pratiques sexuelles sont dès lors pensées comme pratiques réalisant performativement l'identité sexuelle en ce qu'elles citent les normes qu'elles réalisent ou auxquelles elles se conforment. Il faut ici penser que toute pratique sexuelle est citation de la norme qu'elle réalise. Accomplissant une quelconque pratique sexuelle particulière, j'opérerais une sexuation particulière de mon corps parce que, ce faisant, je citerais la norme qui gouverne et institue cette pratique, et la refonderais à nouveau en m'y soumettant. La pratique sexuelle n'est donc que répétition d'une norme qui se donne comme fondée en autorité. Par-là la pratique s'auto-fonde et fonde l'identité qu'elle réalise. Ou dit encore différemment, la pratique s'autofonde en se réitérant constamment. De telle sorte que l'identité sexuelle qui en résulte se trouve fondée du fait même qu'on exécute les pratiques correspondantes.

Cependant, cette itérabilité est structurellement marquée par l'incomplétude : être itérable, c'est être toujours susceptible de se répéter et la réalisation de ce qui est itéré n'est donc jamais terminé. C'est pourquoi l'identité sexuelle ne doit cesser d'exécuter les pratiques correspondantes - la performativité ne doit jamais cesser de se réaliser. Il me faut constamment soutenir mon identité sexuelle en la répétant, sans quoi je risque de la perdre. Cela veut dire aussi qu'elle est fragile est toujours susceptible d'être remise en cause si elle n'est pas répétée ou si elle est répétée différemment - et c'est là que la subversion chère à Butler intervient. En même 
temps, en effet, que l'identité sexuelle trouve dans l'itérabilité des pratiques performatives son fondement, elle y trouve aussi son caractère instable, qui peut toujours lui être révélé. Tel est l'objet des pratiques subversives (parodies, dragqueens, performances diverses) selon Butler : en parodiant les normes qui se réalisent, les dragqueens, par exemple, mettent au jour leur caractère non-naturel et leur fragilité ; ils empêchent les normes de se réaliser en toute quiétude en interrompant leur répétition auto-fondatrice. Stoppées dans leur élan, celles-ci perdent leur fondement et vacillent. Ne reproduisant plus constamment les pratiques sexuelles qui amènent au jour la sexuation des corps en deux catégories, je remettrai ainis en cause les identité sexuelle mâle et femelle et, en conséquence, les corps sexués. On comprend là qu'une des conditions de réussite de la performativité, sinon sa condition de réussite, c'est sa prise au sérieux. ${ }^{9}$ Et que dés lors que leur prise au sérieux, leur non-remise en question qui est l'origine même de leur fondement est stoppée, alors les normes perdent automatiquement leur efficacité. C'est pourquoi, selon J. Butler, il suffit de subvertir une norme, notamment une norme sexuelle, en en jouant, pour remettre question les corps sexués qu'elles réalisent performativement. Il est ainsi toujours possible de faire valoir l'arbitraire de la norme et des réalités sexuelles qu'elle détermine et de réouvrir les possibles que sa naturalisation masquait.

Si la caractérisation de la performativité comme citationnalité permet alors à Butler de réintroduire une liberté d'action au sein même de la réalisation normative, offrant par là un échappatoire aux sexualités "déviantes" qui peuvent toujours réussir à subvertir la sexualité normale naturalisée et constuire d'autres corps sexués, je voudrais néanmoins rappeler que la performativité, telle qu'elle a été conceptualisée à l'origine par Austin, a ses propres conditions qu'il convient de prendre en compte avant de conférer au langage, notamment normatif, un tel pouvoir.

\section{Définition et conditions de la performativité selon Austin.}

Il convient, pour bien comprendre le concept de performativité, de saisir la réalité qu'il désigne. Cette réalité dérive de la découverte que la parole ne se réduit pas à la signification mais est aussi une action. La découverte de la performativité est en effet liée à la découverte du fait que la parole se modélise sous forme d'actes de parole et ne s'efface pas devant ce qu'elle dit. La performativité est la caractéristique propre des actes de parole qui réalisent la réalité qu'ils disent, à la différence des actes de parole qui présupposent une réalité qu'ils décrivent ou sur laquelle ils agissent d'autres façons. Austin va révéler ce phénomène dans plusieurs cas très ordinaires :

\footnotetext{
${ }^{9}$. Cette thématique est elle aussi directement issue de J. D errida.
} 
Toutes les énonciations que nous allons voir présenteront comme par hasard des verbes bien ordinaires, à la première personne du singulier de l'indicatif présent, voix active. Car on peut trouver des énonciations qui satisfont ces conditions et qui, pourtant,

A) ne «décrivent», ne «rapportent», ne constatent absolument rien, ne sont pas «vraies ou fausses»; et sont telles que

B) l'énonciation de la phrase est l'exécution d'une action (ou une partie de cette exécution) qu'on ne saurait, répétons-le, décrire tout bonnement comme étant l'acte, ou «seulement»l'acte, de dire quelque chose.

\section{[...] E x emples:}

(E.a) «O ui, je le veux (c'est-à-dire je prends cette femme pour épouse légitime)» ce «oui» étant prononcé au cours de la cérémonie de mariage.

(E.b) «Je baptise ce bateau le Q ueen E lizabeth» - comme on dit lorsqu'on brise une bouteille contre la coque.

(E.c) «e donne et lègue ma montre à mon frère» - comme on peut lire dans un testament.

(E.d) «Je vous parie six pence qu'il pleuvra demain» (H T D , pp. 5-6/ 40-41 - trad. modifiée)

D ans tous ces cas, l'énoncé prononcé ne renvoie pas à une réalité préexistante, mais crée même la réalité dont il parle ; il l'amène au jour : c'est en cela qu'il est une action. Il est réalisation de quelque chose qui n'était pas là avant son énonciation.

«Pour ces exemples, il semble clair qu'énoncer la phrase (dans les circonstances appropriées, évidemment), ce n'est ni décrire ce qu'il faut bien reconnaître que je suis en train de faire en parlant ainsi, ni affirmer que je le fais: c'est le faire.» (H T D , p. 6/ 41)

Austin est très clair : dire ce type d'énoncé, ce n'est pas décrire ce qui est dit - c'est le faire.

Aucune des énonciations citées n'est vraie ou fausse: j'affirme la chose comme allant de soi et ne la discute pas. On n'a pas plus besoin de démontrer cette assertion qu'il n'y a à prouver que «Damnation !» n'est ni vrai ni faux: il se peut que l'énonciation «serve à mettre au courant» - mais c'est là tout autre chose. Baptiser un bateau, c'est dire (dans les circonstances appropriées) les mots «Je baptise... » etc. Quand je dis à la mairie ou à l'autel, etc., «O ui, je le veux», je ne fais pas le reportage d'un mariage, je me marie.» (H T D , p. 6/41)

Une promesse est l'exemple par excellence de ce que Austin appelle un performatif, c'està-dire un acte de parole qui réalise ce dont il parle. Il en va de même pour le pari ou le baptème : en prononçant l'énoncé de baptème, je fais ce que je dis - je fais parce que je dis et par ce que je dis. C'est pourquoi un énoncé de ce type ne peut pas décrire quelque chose ou être vrai de quelque chose : ce quelque chose n'est pas là avant la réalisation de cet énoncé. Les propriétés 
sémantiques de ce type d'énoncé importent donc assez peu à sa réussite puisqu'elles ne déterminent pas l'action qu'il réalise.

Par conséquent, les conditions de réussites de ces énoncés ne sont pas, ou pas simplement, ses conditions de vérité (l'énoncé n'est vrai de rien du tout, il réalise quelque chose), ce sont plutôt des « conditions de félicité ». Et ces conditions de félicité peuvent se ramener au respect d'un ensemble de procédures énumérées comme suit :

(A.1) Il doit exister une procédure conventionnelle, reconnue et acceptée, qui a un certain effet conventionnel, et qui doit comprendre l'énoncé de certains mots par certaines personnes dans certaines circonstances

(A.2) il faut que, dans chaque cas, les personnes et les circonstances particulières soient celles qui conviennent pour qu'on puisse invoquer la procédure en question.

(B.1) la procédure doit être exécutée par tous les participants, à la fois correctement et

(B.2) intégralement. (H T D , p. 15/ 49)

A la lecture de ces conditions, on comprend bien que ce qui conditionne avant tout la réussite d'un énoncé performatif, ce n'est pas une caractéristique interne ou structurale, comme le pense J. D errida, mais des conditions "externes" à la parole, des conditions non-linguistiques, et pour tout dire contextuelles et sociales. On peut en effet considérer que Austin ramène la réussite d'un énoncé performatif à sa conformation à un rituel socialement accepté dans des circonstances données. L'énoncé performatif est cet énoncé qui, par une sorte de définition conventionnelle, est socialement reconnu comme réalisant une certaine chose comme son effet objectif. L'énoncé performatif demande donc à être reconnu comme respectant cette procédure conventionnelle pour se réaliser véritablement : l'interlocuteur doit reconnaître que je lui fais une promesse, ou comprendre mon énoncé comme promesse, pour que cet énoncé se réalise comme promesse. $^{10}$

Mais cette reconnaissance n'est cependant accordée que si l'énoncé se prête à une telle réalisation dans un contexte donné : je ne baptise pas un navire si je ne suis pas capitaine, comme je n’ordonne pas à des soldats de rompre les rangs si je ne suis pas leur supérieur. Les circonstances doivent être appropriées à la bonne réalisation du performatif. $\mathrm{Ou}$ encore : un

\footnotetext{
${ }^{10}$. Pour plus de détails sur la reconnaissance ou la compréhension nécessaire à l'advenue de l'acte de parole, je renvoie aux travaux de J. Hornsby, par exemple «Feminism in Philosophy of Language : Communicative Speech Acts », in M. FRICKER \& J. HORNSBY, The C ambridge C ompanion to F eminism in Philosophy, Cambridge University Press, 2000, p. 87-106, et de C. Travis, notamment Saying and U nderstanding : a G enerative Theory of Illocutions, New York University Press/ Basil Blackwell, Oxford, 1975 et The U ses of Sense : W ittgenstein's Philosophy of L anguage, O xford University Press, «Clarendon Press », Oxford, 1989.
} 
énoncé performatif ne réussit que s'il est pertinent. Ce qui se décide en contexte. Cela entraine que la force performative résulte toujours d'une reconnaissance sociale contex tualisée.

D errida et Butler sont d'accord avec cette idée puisqu'ils considèrent qu'un énoncé ne cesse de se répéter et donc de prendre effet dans des contextes nouveaux. Mais Derrida fait de cette contextualisation une caractéristique contingente dépendant d'un trait structural de l'énoncé performatif : son itérabilité, conçue comme citationnalité. Pour Derrida, d'une certaine façon, le performatif réussit toujours dans sa performativité, quel que soit le contexte, car ce pouvoir performatif est le propre de la performativité (et les échecs seront eux-mêmes déterminés par cette caractéristique structurale : l'énoncé performatif, étant toujours marquée par cette itérabilité qui le fonde, sera toujours partiel et donc toujours incomplet dans sa réussite ; de telle sorte que l'échec est aussi une caractéristique structurale de l'énoncé : un performatif qui réussit est un performatif qui ne réussit que partiellement.) Ce que dit Austin, bien au contraire, c'est qu'on ne peut pas prévoir la recontextualisation à partir des traits structuraux de l'énoncé, même si celui-ci demande pour s'accomplir d'être contextualisé. L'énoncé, en lui-même, ne demande pas à être recontextualisé, mais il n'est alors capable de rien : l'énoncé "je promets" a bien, hors contexte, certains traits linguistiques qui ne permettent pas, pour autant, de prévoir ce que va dire et faire cet énoncé une fois contextualisé. C'est pourquoi sa réalisation en tant qu'acte nécessite un contexte qui va déterminer cet acte. Par conséquent, c'est, selon Austin, la contextualisation qui détermine véritablement la réussite d'un énoncé, en entrainant, ou non, sa reconnaissance. On ne peut donc prévoir a priori si l'énoncé va se réaliser. Il faut pour cela qu'il satisfasse à des conditions dont on ne peut pas savoir à l'avance quelles elles seront dans un contexte d'utilisation donné. Alors que pour Derrida, on sait a priori que le performatif fonctionne parce qu'il sera réitéré - parce que son efficacité résulte de sa réitérabilité -, il faut pour Austin que tout énoncé soit recontextualisé pour qu'il ait une chance d'accomplir ce que la force performative qu'il détient est censée accomplir. Il faut alors toujours accomplir à nouveau la procédure conventionnelle qui détermine sa force car c'est le contexte qui va déterminer le respect, ou non, de cette procédure. ${ }^{11}$ Et c'est la réalisation en contexte qui permettra d'évaluer si l'énoncé est réussi ou raté. Le performatif ne s'impose donc pas de par lui-même, en raison d'une quelconque propriété structurale.

Or, si les choses se passent telles que Derrida les présente, c'est-à-dire dès lors qu'on comprend cette itérabilité structurale comme citationnalité, alors il y a une circularité dans le raisonnement derridien, que les explications données par Austin permettent de contrer. Le fait de citer quelque chose est en effet déjà un acte de parole particulier, qui nécessite donc lui-même des

\footnotetext{
${ }^{11}$.Je renvoie encore aux travaux de C. Travis, op. at.
} 
conditions particulières pour se réaliser. Ainsi, à supposer qu'un performatif soit une citation de lui-même, on ne voit pas en quoi par cela il réaliserait quoi que ce soit : par définition, l'acte de parole citationnel rapporte quelque chose et ne réalise pas la chose rapportée. Une des conditions de réussite de la citation est en effet la conformité avec ce qui est rapporté, qui doit donc déjà être présent préalablement à la citation. L'acte de parole qu'est la citation rentre donc dans la classe des descriptifs ou des "constatifs", pour reprendre la caractérisation d'Austin. Je peux citer n'importe quelle phrase prononcée par Napoléon, ou même le code Napoléon, je n'amène pas pour autant au jour ce que je cite ; bien au contraire, une condition de la citation implique que ce qui est dit dans la citation ait existé préalablement à celle-ci et la citation ne le réalise pas : par définition, je ne fais une citation que si je répète ce qui est déjà là (c'est même la forme par excellence de la neutralité verbale) ; sinon, je fais un autre acte de parole. Par conséquent, la citation n'amène rien au jour et dire que la performativité réalise quelque chose en se citant ellemême, c'est réduire la réalisation performative à la répétition verbale. Et on ne voit alors pas en quoi cela pourrait influer sur la construction de l'identité sexuelle. A supposer même que le performatif fonctionne comme citation d'une norme, et non pas d'un simple discours, il convient de se rappeler que citer une norme n'est pas la même chose que l'édicter - que ce demier acte nécessite justement d'autres conditions que la citation pour advenir. Pour le dire encore autrement, ce n'est pas simplement à répéter une norme qu'on la réalise. Encore faut-il avoir le pouvoir de l'amener à réalisation, c'est-à-dire remplir les conditions qui en feront un véritable performatif. C'est-à-dire réaliser autre chose qu'une citation.

Parmi ces conditions, figurera la reconnaissance accordée à celui qui fera l'énoncé en tant que fondé à accomplir quelque chose par son moyen. On admet ainsi qu'il utilise correctement et dans les bonnes circonstances la procédure conventionnelle appropriée. Autrement dit, c'est seulement si l'on reconnait à celui qui réalise un performatif le pouvoir ou l'autorité de le faire que le performatif sera réussi. Pour reprendre les caractérisations austiniennes, c'est seulement si la norme est reconnue comme douée d'autorité, c'est-à-dire seulement si, dans ce contexte et dite par cette personne, elle est reconnue comme normative, qu'elle va se réaliser. Pour développer, c'est dire que cette norme doit être un acte de parole "exercitif", celui qui exerce son pouvoir sur la réalité pour la déterminer subéquemment. C'est-à-dire un acte de parole qui porte l'autorité de celui qui le dit à établir dans la réalité ce qu'il dit quant à ce qui est, ou ce qui doit être. C'est dire que ce type d'acte de parole, ce type de performatif, nécessite de satisfaire des conditions très particulières qui ne sont pas remplies par sa simple énonciation. Il faut impérativement que celui qui l'utilise soit, dans le contexte d'utilisation, doté d'autorité et que cette autorité lui soit reconnue pour qu'il réalise pleinement cet acte de parole, c'est-à-dire pour que celui-ci réalise sa 
force performative. Tel est alors le bon usage de la "procédure conventionnelle" décrite auparavant dans ce cas précis - qui n'est pas le même usage que dans le cas d'autres types d'actes de parole, qui eux peuvent évaluer, rapporter des faits, décrire, louer, blâmer, etc. (à chaque acte de parole, pourrait-on dire, sa dimension performative - ce que Austin requalifiera par après de "valeur illocutionnaire"). Comme le dit Austin :

La classe des exercitifs renvoie à l'exercice de pouvoirs, de droits ou d'influences. A titre d'exemple : effectuer une nomination, voter, commander, exhorter, conseiller, avertir, etc. [...] Il y a exercitif lorsqu'on formule un jugement (favorable ou non) sur une conduite ou sur sa justification. Il s'agit d'un jugement sur ce qui devrait être plutôt que sur ce qui est : on préconise ce qui devrait être plutôt qu'on apprécie une situation de fait. Il s'agit d'un arbitrage plus que d'une appréciation, d'une sentence plus que d'un verdict [...] Les exercitifs peuvent impliquer que d'autres sont "obligés", "ont le droit" ou "n’ont pas le droit" d'effectuer certains actes. (H T D , p. 150-154/ 154-157)

Si l'énumération d'Austin reste assez floue, on comprend bien que c'est dans cette catégorie que devrait rentrer le performatif qui, selon Butler, détermine la réalisation de l'identité sexuelle, puisque c'est un acte de parole qui a le pouvoir d'amener au jour ce qui doit être (la norme sexuelle). Cependant, on le voit, une des caractéristiques essentielles de ce type d'acte est d'avoir des conditions sociales de réalisation particulières qui nécessitent que celui qui l'éxécute soit doté de pouvoir. Mais ce pouvoir peut-il véritablement s'appliquer à la réalité sexuelle?

\section{Possibles et impossibles performatifs}

Une fois que l'on a bien compris les conditions de réalisation de la performativité relative à un acte de parole exercitif, il convient alors de se demander les domaines où elle est susceptible d'intervenir. Butler nous dit que la construction de la réalité sexuelle est performative en ce sens qu'elle est advenue d'une sexualité en fonction de normes préalables qui l'établissent. Les normes réalisent performativement la sexualité d'un individu, lui conférant par là une identité sexuelle. Nous comprenons maintenant que cela suppose que ces normes sont dotées du pouvoir de se faire valoir et nécessite qu'elles soient donc pensées comme des actes de parole exercitifs qui ont leur conditions propres.

Il y a alors immédiatement plusieurs choses à souligner : d'une part, Butler ne cesse de confondre réalité sexuelle et identité sexuelle, le donné biologique et la construction identitaire d'un sujet sexuel. Dans l'optique hyper-constructiviste qui est la sienne, cela peut se comprendre, mais cela lui permet surtout d'amalgamer un niveau "symbolique" et un niveau "biologique" en 
une seule unité : le corps sexué. ${ }^{12}$ Il ne s'agit pas de nier que le symbolique et le corporel puissent être très intriqués, mais il convient néanmoins de remarquer qu'on ne joue pas sur les deux niveaux par les mêmes moyens. Et il convient de noter que la reconnaissance nécessaire à l'advenue du performatif fixe un domaine d'activité relativement restreint à celui-ci : le domaine symbolique. Le performatif est un produit d'une reconnaissance socialisé qui ne peut naturellement pas agir directement sur autre chose que le domaine institué par le jeu de cette reconnaissance social. Il peut bien sûr à terme entrainer une intervention sur les corps (en tant que je me conforme à une norme qui guident des interventions chirurgicales, par exemple), mais son action n'est pas immédiatement corporelle : faire une promesse, ce n'est pas mettre au jour une réalité physique, de même que donner un ordre. Dès lors la réalité sur laquelle joue le performatif est une réalité linguistique/ symbolique : c'est un acte de parole, qui est bien distinct d'un acte physique. ${ }^{13}$ Aussi, s'il est logiquement possible, de jouer sur le niveau symbolique en utilisant le langage et sa performativité, il nous semble que c'est un pêché qu'affectionne le poststructuralisme que de croire que la réalité biologique/ corporelle elle-même peut résulter d'une action linguistique/ symbolique. Or c'est bien ce que fait Butler quand elle considère que les normes se réalisent biologiquement de façon performative.

Elle ne le dit pas toujours aussi directement mais hésite en fait entre deux approches bien distinctes $^{14}$. Soit elle considère, en s'approchant alors des observations faites avant elle par C. Delphy $^{15}$, ou par C. Guillaumin avec son concept de "sexage" ${ }^{16}$, que la sexualité est "arraisonnée" par des normes qui dès lors construisent les identités sexuelles à partir de certaines caractéristiques arbitrairement retenues comme importantes. Ce qui peut se lire : il y a une utilisation politique à visée inégalitaire d'une définition de l'identité sexuelle en fonction de caractéristiques biologiques prises arbitrairement (la capacité à mettre les enfants au monde est généralement l'élément qui sert à poser une distinction). Il y a alors une construction arbitraire des catégories de "sexe" à visée politique.

$\mathrm{Si}$ là Butler veut seulement remarquer que tout processus de dénomination, et notamment de la dénomination d'une sexualité comme hétérosexuelle ou homosexuelle, est arbitraire, elle ne fait jamais que redécouvir ce que disait Locke dans son E ssai sur l'entendement humain, quand il remarquait que les noms d'essence ne renvoient pas à une réalité et ne recouvrent jamais qu'un conglomérat d'idées plus ou moins confuses et plus ou moins

\footnotetext{
${ }^{12}$. A cette fin, elle utilise naturellement Lacan, mais notre propos n'est pas de critiquer l'usage qu'elle en fait.

${ }^{13}$. D eux indices de cela : la simple description des événements physiques (acoustiques) ne permet pas de rendre compte de l'acte de parole réalisé ; la détermination de celui-ci ne peut se faire que sur un arrière-plan de pratiques.

${ }^{14}$. D ans ses textes, elle alteme constamment un discours sur le "sexe" et un discours sur le sexe.

${ }^{15}$. Voir C. D elphy, L 'ennemi prindipal, tome 2, Syllepse, Paris, 2000.
} 
cohérentes. Butler découvre donc l'arbitraire du signe et des définitions conceptuelles. Et on peut certainement faire un usage idéologique et discriminant de ces définitions. Pour autant, nommer quelque chose, ce n'est pas le créer ; l'acte de parole de la dénomination, dont Austin a étudié toutes les subtilités dans "Comment Parler"17, présuppose au contraire une réalité préexistante ; telle est une de ses conditions de félicité. Si je nomme quelque chose qui n'existe pas, alors mon acte de parole échoue. Butler veut donc bien plutôt considérer que toute dénomination est une définition performative : elle réaliserait ce qu'elle donnerait comme naturel et prétendrait décrire.

Tout dépend alors du rôle qu'elle fait jouer aux normes : considérer qu'elles définissent et réalisent une identité sexuelle en tant qu'identité socialement définie n'est pas absurde, ni impossible, puisque certaines normes ont le pouvoir de s'imposer comme représentant les identités sexuelles "naturelles", sous certaines conditions (notamment à condition d'être dotées de l'autorité de se réaliser, si elles ont valeur de loi, ou de textes médicaux). Mais on en reste alors au niveau symbolique des rôles sexuels, c'est-à-dire des activités sexuelles considérées comme définitionnelles d'un sexe, et on ne s'éloigne pas énormément d'une problématique du genre qu'on ne fait qu'étendre. On dit seulement que la délimitation définitionnelle des sexes est socialement arbitraire et renvoie en fait à des normes qui s'imposent comme définitionnelles. Ce qui est donc créé performativement dans ce cas, ce ne sont jamais que les identités sexuelles sociales, ou les rôles sexuels socialement définis qu'adoptent les corps en croyant pratiquer une sexualité donnée comme naturelle. Mais ce n'est pas dire que la sexuation des corps dérive de la performativité des normes. On dit seulement que des corps déjà sexués adoptent des identités sexuelles socialement constituées et définies en réalisant telle ou telle pratique. Cela suffit pour penser tous les jeux de pouvoirs imaginables : la définition sociale des identités sexuelles peut conférer telle ou telle valence à telle caractéristique ou à telle pratique, exclure telle autre, condamner une autre encore. De telle sorte que certaines identités sexuelles sont socialement admises parce que considérées comme naturelles (dans notre société, l'homme et la femme). On peut donc admettre que l'identité sexuelle que je crois avoir naturellement n'est en fait jamais qu'une identité socialement construite, c'est-à-dire correspondant à une définition performative de la normalité qui réalise les normes définitionnelles : je ne suis un "homme" que parce que je considère qu'un certain nombre de mes caractéristiques et mes pratiques font de moi un homme. Et ce qui me fait considérer ces caractéristiques et ces pratiques comme définitionnelle de l'homme, ce n'est que la définition performative, parce que socialement accepté et reconnue, des

\footnotetext{
${ }^{16}$. C. Guillaumin, Sex e, race et pratique du pouvoir, Côtés femmes, Paris, 1992.
} 
identités sexuelles. On peut alors comparer ici la définition performative des identités sexuelles à la définition performative du cadre ; comme l'a montré L. Boltanski dans son livre sur les cadres $^{18}$, en effet, ceux-ci n'ont été portés au jour que par la grâce d'une définition sociale d'une nouvelle réalité administrative. Mais celle-ci existe désormais bel et bien dans la réalité sociale et dénomme une certaine identité. Je peux avoir cette identité de cadre, avec les caractéristiques; les devoirs et les droits qui y sont attachés. Et je n'ai cette identité de cadre que parce que je me conforme à la définition administrative du cadre, définition qui dès lors est performative en ce qu'elle fait advenir ce qu'elle nomme. De la même façon, j'ai une sexualité mâle ou une sexualité femelle, parce que je réponds, par un certain nombre de mes caractéristiques, à la définition sociale de l'homme ou de la femme (qui attache des pratiques différentielles à ces identités), faisant advenir cette définition dans la réalité en me conformant aux pratiques qu'elle y rattache. Il semble donc qu'on puisse penser une réalisation performative des identités sexuelles, dès lors que leurs normes ont le pouvoir de se faire advenir.

Mais Butler semble plus souvent aller beaucoup plus loin et dire qu'il y a une construction de la sexualité biologique (du sexe, et pas seulement du "sexe") par sa dénomination implicitement normée. Pourtant le fait de l"arraisonnement" des corps et des pratiques par une définition performative n'entraîne pas une sexuation biologique des corps ; cela veut simplement dire qu'on retient arbitrairement telle caractéristique biologique comme importante pour définir telle identité et comme devant impliquer telle pratique - ce qui construit une identité sexuelle stable à laquelle sont associé des pratiques et des caractéristiques. C'est ce qu'on appelle une prophétie auto-réalisatrice : disant, de manière autorisée, ce que c'est qu'être un homme ou une femme, je crée ce que c'est qu'être un homme ou une femme - je parviens à imposer aux membres d'une société donné cette définition et à la réaliser en faisant en sorte qu'ils s'y conforment. Mais les caractéristiques sexuelles biologiques sont déjà données et l'identité sexuelle que j'adopte, dès lors reconsidérée comme rôle sexuel imposé, se construit sur ces caractéristiques - elle ne construit pas ces caractéristiques. Cette dernière éventualité supposerait que la peformativité du langage agisse directement sur les corps. Or nous avons vu que par définition, l'action performative est une action symbolique, c'est-à-dire que ses conditions de réussite sont essentiellement une condition de reconnaissance sociale qui tient pour accompli une certaine réalité. La promesse, pas plus que l'ordre ou le mariage ne sont des réalisations physiques : ce sont des coups joués dans un jeu social réglé. Pour que la performativité joue sur la sexuation des corps, il faudrait qu'existent des actes de parole avec des conditions de félicité

17. Voir J. L. Austin, "How to Talk", in Philosophical Papers, edited by J.O. Urmson and G .J. Warnock, Oxford University Press, "Clarendon Press », Oxford, 3ème édition : 1979. 
très particulières qui toucheraient au corps biologique. Cela présupposerait une procédure conventionnelle reconnue dont la réalisation entraîne une modification biologique. Or il semble que l'inexistence de cette procédure, aussi implicite qu'elle puisse être, est un constat empirique. Aussi bien, on peut poser qu'il n'y a pas de réalisation performative du corps, ni, par conséquent, de sexuation performative du corps.

Cela conduit à repenser différemment la subversion, à considérer ses conditions et à limiter considérablement son ampleur. Premier point : on ne peut pas subvertir les corps biologiques en empêchant les normes de se réaliser puisque cette réalité biologique ne dépend pas des normes. Qu'en est-il alors de la subversion des identités sexuelles qu'on acquiert en se conformant à leur définition ? On pourrait penser qu'il suffit de ne pas se conformer à leur définition pour les remettre en cause. Qu'il suffit donc d'empêcher la performativité d'opérer. Mais cela n'est pas aussi simple que le laisse penser Butler quand elle considère que cela peut se faire par un simple mouvement de volonté subversif : il ne faut pas oublier les conditions de réalisation de la performativité, qui font que parfois celleci s'impose à nous. Je ne décide pas toujours de ne pas tenir un ordre pour un ordre - l'occasion ne se présente pas toujours de l'ignorer, les circonstances ne permettent pas toujours sa subversion. Il faut comprendre que le contexte possède son objectivité propre qu'il confère à la performativité et que celleci impose bien plus souvent sa reconnaissance qu'elle ne la propose. C'est donc ramener la subversion à ses conditions sociales : ce qu'il convient de faire, c'est modifier les conditions sociales qui déterminent la réussite d'un performatif considéré comme ségrégatif plutôt que le réitérer sur un mode ironique qui n'affecte en rien ses conditions d'efficacité.

\section{Condusion}

Si la pensée critique de J. Butler se restreint aux identités sexuelles en tant qu'elles sont socialement définies, il apparait tout à fait légitime de considérer que celles-ci sont performativement construites par des normes qui ont le pouvoir de s'imposer à la réalité. Il apparait cependant excessif et illusoire de penser que le performatif intervienne dans la construction biologique de l'identité sexuée, étant données ses conditions de réalisation. Il convient donc de restreindre la portée de la théorie de Butler pour en sauvegarder la pertinence : au domaine de la définition des identités sexuelles, lieu symbolique où la définition performative peut toujours laisser place, à terme, à une définition constative, rapportant simplement les faits, qu'ils soient naturels ou historiquement construits.

\footnotetext{
${ }^{18}$. Voir L. Boltanski, L es cadres : la formation d'un groupe social, Minuit, Paris.
} 
Il convient aussi de rappeler que la réussite du symbolique a ses conditions que les zélateurs de la subversion symbolique se plaisent à ignorer : des conditions matérielles et sociales qui ne peuvent être remises en cause par la simple volonté subversive. Une véritable attention aux conditions de fonctionnement des performatifs définissant l'identité sexuelle, une attention au concret de la vie du langage, leur apprendrait que celui-ci est tout à la fois plus affecté par les conditions concrètes des agents et moins «matériel » que ne le pensent les déconstructionnistes. Bref, vouloir changer réellement lidentité sexuelle des personnes, en tant que celleci est injurieuse, restrictive, blessante, amène normalement à affronter les conditions réelles de réalisation des performatifs qui les instaurent et à se confronter aux conditions sociales, économiques, juridiques qui les sous-tendent. Les actes de parole ne sont pas qu'affaire de représentation.

\section{Livres de J. Butler utilisés :}

_ Gender Trouble, Feminism and the Subversion of Identity, Routledge, New York \& London, 2nd Ed. : 1999.

_ Bodies That Matter, On the Discursive Limits of "Sex", Routledge, New York \& London, 1993.

_ Excitable Speech, Politics of the Performative, Routledge, New York \& London, 1997.

Bruno A MBROISE,

Université Bordeaux 3 / IH PST

A paraitre in Critique Sociale, PF N SP, Paris, 2003. 